\title{
Exercising During Pregnancy: researched-based recommendations
}

\section{Abstract}

Exercise offers many benefits to both mother and baby before, during and after pregnancy. It is becoming increasingly accepted that the expectant mother's level of physical health during pregnancy (Morris and Johnson, 2005) will influence their recovery post-partum.

Recent research also supports this hypothesis and official guidelines encourage mothers experiencing low-risk, normal pregnancies, to take part in daily exercise - albeit with modifications to their otherwise regular routine as the foetus grows (AGOG 2015, Clapp, 2006) as the pregnancy progresses More than ever before, the emphasis of musculoskeletal health is in moving pain free and continuing exercise to late on in the term of pregnancy to prevent, reduce and manage discomfort. Many expectant women develop sometimes temporary - so-called upper and lower crossed syndromes (UCS and LCS)- characteristic to the LCS is lumbar lordosis, which contributes to the very high prevalence (<50\%) of low back pain in pregnant women (Wang et al, 2004). Balance may be affected by the changes of the body's centre of gravity (COG), predisposing pregnant women to an increased risk of falling. Another musculoskeletal change during pregnancy is increased ligamentous joint laxity - thought to be secondary to the influence of the increased levels of oestrogen and relaxin. Theoretically, this would predispose pregnant women to increased incidence of strains and sprains (Artal and O'Toole, 2003), lower back pain and pelvic girdle pain (Gutke et al, 2008, Maclennan etal., 1986).

In each and every case, the use of corrective and preventative functional exercises are needed that mimic activities of daily living $(A D L)$ in conjunction with activating the pelvic floor muscles. It is imperative that the pelvic floor regains its strength soon after birth to avoid post-pregnancy complications such as incontinence. Postural changes can also be required to encourage one's posture to return to its pre-pregnancy state and to thus avoid chronic musculoskeletal problems.

Keywords: pregnancy, musculoskeletal health, functional exercise, upper and lower crossed syndrome, pelvic floor

\section{Introduction}

Although every woman and every pregnancy is different, every mother should be able to find an adequate exercise practice that not only helps them maintain a reasonable level of fitness during and after pregnancy (Price et al. 2012), but can keep ailments and pregnancy complications at bay. Research shows that mothers who exercise also feel less tired, have healthier weight gain and experience less leg swelling and back pain. Regular exercise can also reduce labour duration and the likelihood of post-natal depression, while helping new mums to regain their pre-pregnancy figure more quickly. It also reduces the likelihood of gestational diabetes and the development of pre-eclampsia (gestational hypertensive syndrome) (Hopkins and Artal, 2013; Price et al. 2012; Moyer et al. 2015). Women who have been active before pregnancy can continue with physical exercise provided that they discuss with their health care providers and/or fitness professionals how and when it needs to be adjusted to accommodate the growth of the baby in utero. It goes without saying that contact sports - such as martial arts, horse riding and skiing - which involve a risk of impact with the stomach, should be avoided. Those with known pregnancy complications should consult their health care provider before commencing any exercise. In later articles that will follow, focus will be on suggestions on preventative and corrective exercises for each trimester, which I believe will help mothers continue with their chosen recreational exercise.

Equally important to care for the so-called core - or the inner unit - is a series of muscles that act as stabilisers during movement and help to transfer force between extremities, in particular to the transverse abdominis and the pelvic floor muscles. Almost every movement initiated from the core, therefore, it is of paramount importance to stability, strength and whole body integrity. A principle aim of this article is to pose the question of safety in exercise and the promotion of good practice based on current research and guide exercise specialists how to care for their pregnant and post-partum (post- birth) clients.

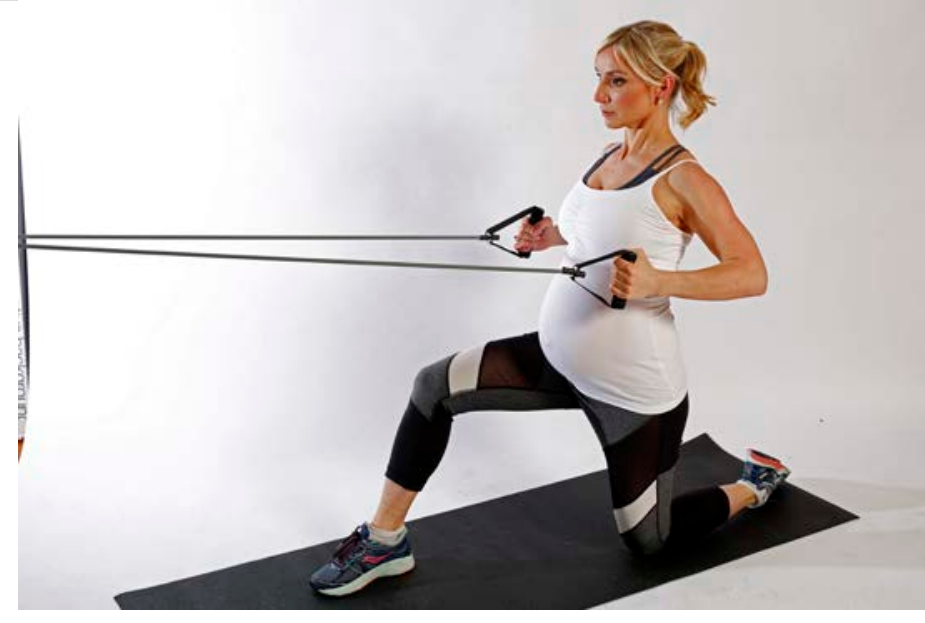

Questions:

Who can and should exercise during pregnancy; what are the benefits and risks and the current exercise guidelines?

What are the anticipated anatomical and hormonal changes during pregnancy and how does it relate to postural changes?

Can excessive postural alignment changes and pregnancy complications be prevented with regular, planned and structured recreational exercise?

\section{The Exercise Guidelines}

Pregnancy is a time of many physical and physiological changes. Not surprisingly, these changes impact the musculoskeletal system, and can present a number of problems. It is currently recognised that habits adopted during pregnancy could actually affect a woman's health not just during her pregnancy, but for the rest of her life (Artal and O'Toole, 2003).

For the first time, official governmental recommendations suggests that exercise playing an important role in the prevention and management of gestational diabetes - a metabolic disorder that typically develops during pregnancy. This form of diabetes is characterized by abnormally high blood sugar levels due to the pancreas failing to produce enough insulin. During pregnan$c y$, the insulin sensitivity of the bodily cells decreases, making it harder for the cells to store sugar. Hence regular exercising in obese and overweight woman who are starting exercise the first time is recommended. It seems non-weight bearing exercises such as swimming and cycling would be the most beneficial and tolerable to the mother and also enough to elicit fetoplacental adaptations. Resistance training - in addition to aerobic exercise - would also bring health benefits to mother and offspring (Hopkins and Cutfield, 2011).

As far the recent history of official exercise recommendations go, in 2002, the American College of Obstetricians and Gynecologists (ACOG), the main regulating body in the United States, published its new recommendations and guidelines for exercise during pregnancy. It was to change the previously held view (based on the 1994 guidelines) entirely on how exercise should be promoted.

Derived from the American College of Sports Medicine (ACSM) guidelines, regular recreational exercise should be promoted for its overall health benefits during pregnancy and in the post-partum period (Artal and o'Toole, 2003). Individuals were encouraged to do 30 minutes or more of moderate intensity physical activity and preferably on most or all days of the week; totalling at least 150 minutes per week (Pate et al. 1995).

Since 2002, the World Health Organisation (WHO) and the ACSM have issued new evidence based recommendations indicating that the benefits of exercise far outweigh the risks on pregnant women (WHO, 2010; Garber et al. 2011). It has been put forward that out of the 150 minutes, healthy adults should also perform resistance exercises for each of the major muscle groups for at least two days a week. These should include sensorimotor exercises, which are known to be promoting balance, agility, and coordination. Furthermore, on more than two days a week, it is recommended that focus should be on maintaining joint range of movement, with a series of flexibility exercises for each the major muscle-tendon groups (Garber et al. 2011).

The newest ACOG and RCOG (Royal College of Obstetricians and Gynaecologists) guidelines (2015) also promote exercise for those who have not been previously exercising, as well to those with medical or obstetric complications, (but only after thorough medical evaluation). The revised RCOG guidelines also now suggest $45-60$ minutes of moderate exercise on most days, which could include 10-20 minutes of warm up and cool down stretches. The guidelines also note that instead of using the heart rate $(H R)$ as a guide to what constituted moderate intensity to the individual - a corresponding HR was set on or under $140 \mathrm{bpm}-$, it is advised to use perceived exertion as 
a measure. The BORG Scale of Perceived Exertion is a talk test, during which the exercising pregnant woman should be able to carry on a conversation, which on a scale of 6-20 of exertion, should be perceived as somewhat hard rated as 12-14 on the scale, exercises should not be progressed to absolute muscle fatigue. Figure 1.

\section{Furthermore the recommendations state:}

- During pregnancy, aerobic and strength conditioning exercise is considered to be safe and beneficial.

The aim of recreation reach peak fitness.

- Pelvic floor exercises during pregnancy and immediately after birth may reduce the risk of urinary and faecal incontinence (lack of voluntary control over urination or emptying the bowels) in the future.

- For most women, it is safe to exercise as soon after birth as one feels ready; taking into consideration delivery (an uncomplicated, vaginal birth will allow women to do gentle exercises almost right away).

- Pelvic floor exercises could be resumed in the immediate post-partum period.

Women who do strength and conditioning exercise during pregnancy tend to have a shorter labour time and fewer delivery complications. It is therefore an encouraged form of exercise, however professional supervision might be necessary.

Figure 1. Further recommendations by ACSM and AGOG

Recreational exercise refers here to any kind of energetic exercise (aerobic) (such as swimming, running or even walking) and strength conditioning exercise that consist of planned, structured and repetitive bodily movements done to improve one or more component of fitness (ACSM, 2008). Aerobic exercise is any activity that stimulates a person's breathing and blood circulation (beyond that of resting), and the employment of large muscle groups in a continuous and rhythmic manner. Performing it two to four times a week has been shown to help improve or maintain physical fitness and there is no evidence of harm to the foetus when not contraindicated in pregnant women. However, studies that showed improvements in cardiovascular fitness due to aerobic activity are small and have not been able to offer satisfactory conclusions as to what other effects on mother and baby it might have. Larger, better trials are still required to be undertaken (Kramer and McDonald, 2006].

This uncertainty that prevails to date may contribute to the low percentage $(16 \%)$ of pregnant women reported to be exercising according to these guidelines (Rousham et al, 2006). The lack of physical activity during pregnancy may also be due to a lack of clear guidance, inconsistent motivation, advice, concerns about maternal and fetal injury, and insufficient support from health care providers (Rousham, 2006). A significant contributing factor could be, which is indicated from feedback from obstetricians and GPs, that a significant portion never discuss exercise or physical activity with their pregnant patients (Entin \& Munhall, 2006, Herring et al, 2010); and doctors in obstetric training programs are not provided with much information about exercise in pregnancy. Most worryingly, there appears to be a trend to classify women in the wrong body mass index (BMI) and weight categories, and therefore a major lack on advice to patients to keep moving and exercising regularly. Added to this, the majority of women tend to underestimate their body mass index (BMI) category and overestimated the weight gain recommended during a healthy pregnancy (Shub et al. 2013). Certainly, more reliable data is warranted to provide a clinically significant, evidence-based exercise regimen for pregnant women in general that will promote consistent and confident use by clinician and patient alike. It also seems evident that as women reach the third trimester, the physical limitations and the rapidly growing foetus accompanies a decrease in their activity level (Rousham et al, 2006). Increasing exercise load in the third trimester is not necessary as the growing weight of the foetus will provide the increased resistance.

Strength resistance training as part of a conditioning program has been studied although not extensively. In one study, individually prescribed strength training (one set of up to 12 repetitions for beginners) of multiple muscle groups were used for pregnant women.

Foetal HR were monitored during training to decide whether the activity changed the foetus's HR at 28 and 38 weeks gestation, they remained unchanged. It was concluded that relatively low weights with multiple repetitions (using an intensity less than 70\% 1RM - 10 reps or more per set) lifted through a dynamic range of motion appear to be safe and effective type of resistance exercise.

General advice for intermediate and advance clients are 2-3 sets of functional exercises for each major muscle group in an integrated fashion for 12-15 repetitions (Schoenfeld, 2011, Baechle et al, 2008). Firstly, emphasis should be placed on training the core musculature with functional weight-bearing exercise, which can counteract lumbar region discomfort and alleviate symptoms with lower back pain (Hopkins and Cutfield, 2011).

Secondly, to optimise functional ability, exercises should be multi-planar in focus; free weights, cables, bands and body weights can be employed. It would also be prudent to limit repetitive isometric or heavy resistance weightlifting and stop any exercise that results in pressor effect (increased arterial blood pressure) and increased abdominal pressure, which can be potentially dangerous on the fetus.

\section{Physiological and morphological changes in the body}

Pregnancy is a unique time in the life of a woman with many physiological, morphological and hormonal changes affecting the musculoskeletal system. Many of the biomechanical changes occur in the pelvis or in adjacent joints. Kinematic changes in late pregnancy are related to increased joint laxity, hormonal changes, decreased or increased range at the hip joint and change in the COG.

The hormone relaxin plays the most decisive role in the mechanics of movement, as it provides greater ligament laxity in the pelvis and on the peripheral joints (Butler et al. 2006; Calguneri et al. 1982; Schauberger et al. 1996), remodeling of multiple tissues of the musculoskeletal system. For example, there is the need of the widening and increased mobility of the sacroiliac joints and pubic symphysis in preparation for the fetus' passage through the birth canal (Dehgham et al. 2014) but this loosening effect isn't limited to these joints only, it's effect is more systematic: as such joint laxity in the anterior and posterior longitudinal ligaments of the lumbar spine creates more instability in this region and predisposes it to muscle strain (Figure 2). Figure 2: Excessive lordotic curve (adapted from the book: Fixing You: Back Pain During Pregnancy)

Studies have also suggested a rela-

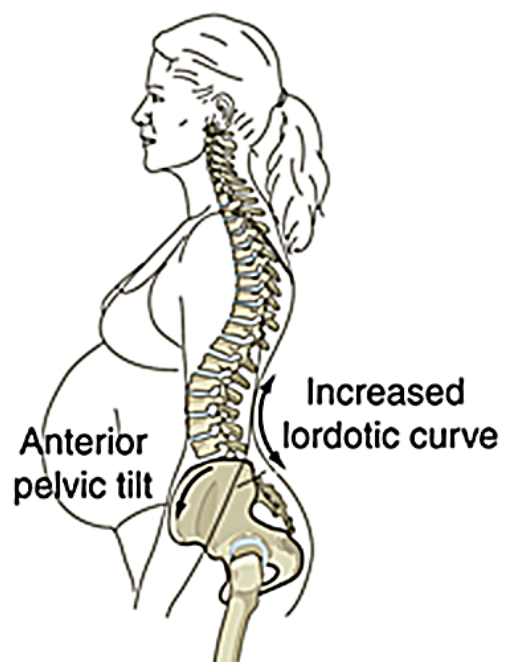

tionship between higher relaxin and progesterone serum levels in pregnant females with pelvic girdle pain syndrome (Maclennan et al. 1986) and pelvic floor dysfunction (Harvey et al. 2008).

There will be a new position of the pelvis in late pregnancy seems to be a consequence of the increasing weight of the uterus, placenta, and fetus. As the displacement of the COG moves upward and anteriorly (Foti et al. 2000; Rodacki et al. 2003; Whitcome et al. 2007) it gradually drives the pelvic tilt further anteriorly of approximately 5 degrees (Foti et al.2000; Hagan et al. 2010) (Figure 3), which in turn enables a newly found control of the COG (Foti et al. 2000). This position weakens the rectus abdominis's and the transversus abdominis's capacity to produce force and facilitate smooth force transfer as they are stretched both by the anterior tilt and the outward stretch of the abdominal wall due to the growth of the pregnancy bump.

The greater participation of the abductors (piriformis, gluteus medius) and extensors muscles (hamstring, lower back muscles) attached to the hips can make them overactive, hence can contribute to lower back, pelvis and hip issue (Foti etal; Huang etal, 2002) (Figure 4).

Figure 3: increase anterior pelvic tilt (source: www.theptdc.com)

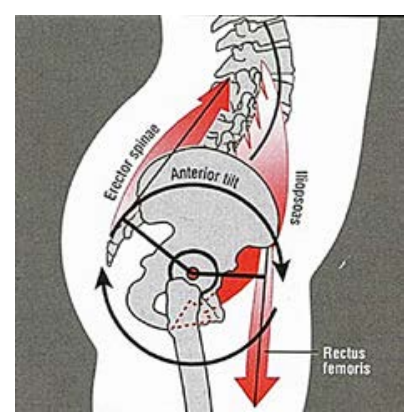

Two visible kinematic changes to the musculoskeletal system are the external rotation of the hips during gait (feet will turn out) and to maximize safety during motor tasks such as walking the widening of the step (Forczek and Staszkiewicz, 2012). In fact, these changes are happening because of the increased medial-lateral instability of the mother as her pregnancy progresses, this consequence seems to have great significance. These changes significantly affect the balance and body's stability (Dunning et al. 2003; Butler et al. 2006), and not surprisingly, the risk of falling during pregnancy is 


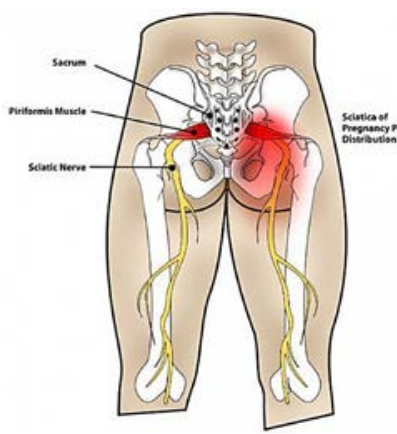

almost matched by the probability of it having in the elderly aged $>65$ years (Kaname et al. 2015). Most falls has been reported in the second trimester (Dunning et al. 2003) and these statistics also illustrate the importance of fall prevention that can be achieved by single leg balance and coordination multi-plane and multi-directional exercises.

Figure 4: overactive piriformis, known to cause sciatica in pregnancy (source: www. healdove.com)

Other factor that heavily influences the musculoskeletal system is the rapid increase in maternal weight. When weight is in excess (Figure 5), it may cause several adverse effects, including gestational hypertension, gestational diabetes, difficulties during labour and weight retention post-partum. The increased weight in pregnancy may significantly increase the forces across joints such as the hips and knees by as much as $100 \%$ (Karzel and Friedman 1991) during weight bearing exercises, and so caution is required when carrying lots of excess weight. A strategy to control excessive weight gain (as well as having a structured diet before and during pregnancy) is the performance of structured recreational physical activity that can range from aerobic activities to resistance and circuit training. Walking is highly recommended for overweight and obese pregnant women if beginners to exercise.

Figure 5. Normal weight gain during pregnancy table (adapted from the In stitute of medicine)

\begin{tabular}{cc}
\hline Pre- pregnancyBMI & Total weightgain in $\mathrm{Kg}$ \\
\hline Underweight $\left(<18.5 \mathrm{~kg} / \mathrm{m}^{2}\right)$ & $12.5-18.0$ \\
Normal weight $(18.5-$ & $11.5-16.0$ \\
$\left.249 \mathrm{~kg} / \mathrm{m}^{2}\right)$ & \\
\hline Overweight $\left(25.0-29.9 \mathrm{~kg} / \mathrm{m}^{2}\right)$ & $7.0-11.5$ \\
Obese $\left(>30.0 \mathrm{~kg} / \mathrm{m}^{2}\right)$ & $5.0-9.0$ \\
Twin pregnancy & $15.9-20.4$ \\
\hline
\end{tabular}

\section{Pregnancy posture}

In static postural assessments, it is best to assess the clients in static lateral view. The most common changes to the posture are exaggerated lordotic curve of the lower back, excessive forward flexion of the neck (protruding neck); forward movement of the shoulders; locked out knees typically occur to compensate for the enlarged uterus; and the anterior shift of weight and change in center of gravity (Figure 6).

Stretching of the TVA muscle and weakness and separation of abdominal muscles such as the rectus abdominis further impede neutral posture, place even more strain on the small stabilisers such as the paraspinal lower back muscles, the sacrospinalis and the erector spinae group. When these muscles are overactive, the client will feel strain when leaning for-
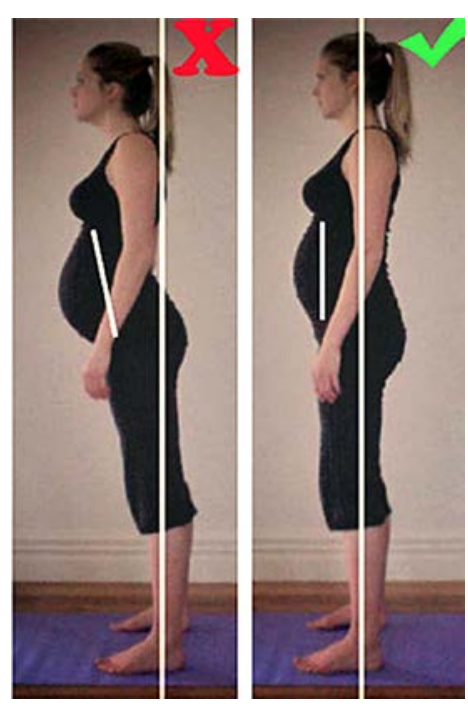

Figure 6

Changes in posture during pregnancy

(source: www.mamalionstrong.com) ward or sitting for prolonged periods of time.

In pregnancy and in post-partum, pregnant women can develop muscle imbalance syndromes named after Vladimir Janda (the physician, physiotherapist), called the Janda's lower and upper crossed syndrome (LCS and UCS). Diagnosis of these is often made through observation of posture and certain movement patterns related to pain syndromes (Jull et al. 1999). The UCS is also often referred to as shoulder girdle crossed syndrome - referring to the crossed pattern of the overactive muscles with the counter crossing of the underactive muscles. When viewed from the side, a X-pattern can be seen between two sets of muscles. The tightness of the upper trapezius and the leavtor scapula (neck extensor) at the back crosses with the tightness of the sternocleidomastoid, pectoralis major and minor at the front. Weakness of the cervical flexors anteriorly crosses with weakness of the middle and lower trapezius and rhomboids (Figure 7a).

LCS is a typical imbalance that is linked to the lower back region and is characterized by the imbalance of tight and short hip flexors such as the iliopsoas, the lumbar region extensors and erector spinae group, and the inhibited/weakened gluteus maximus and on the cross side the stretched abdominal muscles. The syndrome promotes the excessive anterior tilt of the pelvis mentioned before and protrusion and weakening of the lower abdomen with that increased lumbar lordosis and a slightly flexed position of the hips (Figure 7b).
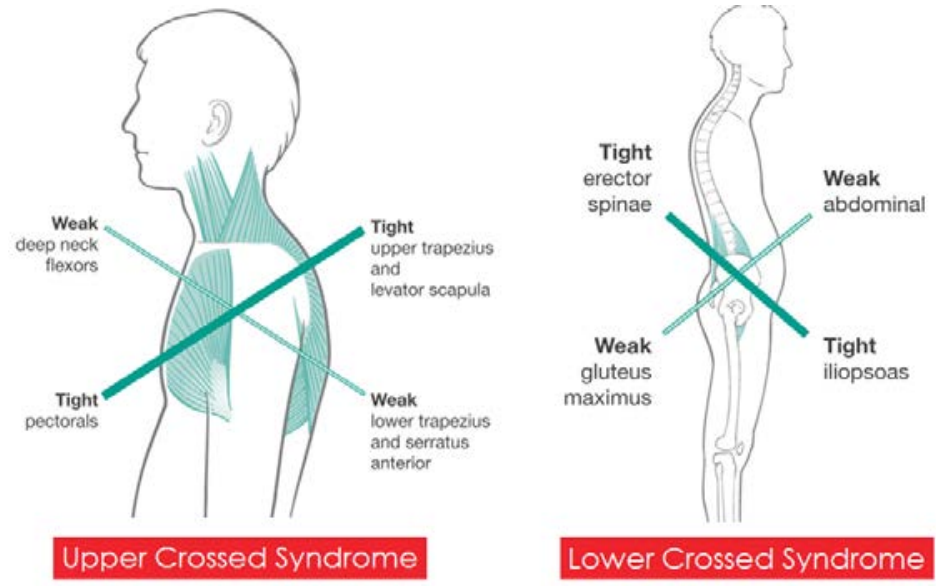

Figure 7: a) Janda's upper crossed syndrome (UCS) and b) lower crossed syndrome (LCS) that can accompany any pregnancy

\section{Conclusions}

Despite the fact that pregnancy is a time for rapid anatomical and physiological changes, exercise seems to have minimal risks to expectant mothers and their foetus, and it is shown to have great benefits to most women. Women with uncomplicated pregnancies or possessing uncomplicated histories of pregnancies should be encouraged to exercise before, during and after pregnancy - even if they have been sedentary before conceiving. It's the role of the obstetric health care provider and the GP to advise mothers-to-be on the optional exercises and modalities and while it may seem that professionals need to update their knowledge to the very latest of exercise guidelines for pregnant mothers, it is crucial that they encourage overweight and obese women to exercise as well to help them avoid pregnancy complications.

Exercise may reduce the risk of gestational diabetes, preeclampsia and caesarean deliveries to mention the more serious pregnancy complications. Shift of the COG, postural changes, changes in balance, the prevalence of the UCS and LCS is evitable, however. Yet with regular, planned functional and conditioning exercises, these side effects can be minimized and pregnancies can be made a more pleasant experience for many women. Additional large control trial researches are still needed into the effects of exercise on certain pregnancy conditions and on the fetus but it seems that a mix of moderate intensity exercises, such as aerobic activities, strength resistance training and flexibility exercises are considered to be safe and are advised to be performed on most days of the week for 45-60 minutes each day. However, as every day is different during pregnancy, expectant mother should listen to their bodies when performing even routine, familiar exercises and alter intensity and frequency to what is comfortable and sensible for them.

Notes: Seek permission from your doctor or midwife before starting exercising. Always ask whether there is any reason why you shouldn't follow a moderate intensity simple exercise program. Always make sure wherever you exercise that the room is well-ventilated, to avoid overheating. Eat a small snack 30 minutes before exercising and after exercise. Stay well-hydrated and drink during workouts. If you experience any pain or discomfort stop and reassess your body's alignments when performing the exercises. If that doesn't help, seek advice from a healthcare professional and stop the exercise. Follow RPE guidelines. Don't exercise if you experiencing any of the following: dizziness or fainting, vaginal bleeding, sudden water discharge, extreme shortness of breath, headaches, signs of labour, decreased fetal movement, chest pain and calf pain or swelling. For more absolute and relative contraindication on aerobic exercise in the pregnant population, please visit the ACOG or RCOG websites (Artal and O'Toole, 2002). 


\section{References}

ACOG Committee. (2002): Opinion no. 267 exercise during pregnancy and the postpartum period. Obstetrics and Gynecololgy. 99:171-3. ACSM's guidelines for exercise testing an prescription. (2008): 2nd ed. Philadelphia (PA): Wolters Kluwer/ Lippincott Williams \& Wilkins. Artal R. and O'Toole M. (2003): Guideline of the American College of Obstetricians and Gynecologists for exercise during pregnancy and the postpartum period. British Journal of anorts Medicine 37(1): 6-12. DO1:10.1136/ bjsm.37.1.6

Baechle T., Earle R. and Wathen D. (2008): Resistance training. In: Essentials of Strength Training and Conditioning (3rd). Baechle T and Earle R, eds. Champaign, IL: Human Kinetics, pp. 381-412.

Branco M., Santos-Rocha R. and Vieira F. (2014): Biomechanics of gait during pregnancy. Scientific World Journal. Online 2014: 527940. doi: 10.1155/2014/527940

Butler E.E., Colón I., Druzin M.L., Rose J. (2006): Postural equilibrium during pregnancy decreased stability with an increased reliance on visual cues. American Journal of Obstetrics \& Gynecology. Oct;195(4):1104-8. DOI 10.1016/j.ajog 2006.06.015

Calguneri M., Bird H.A. and Wright $V$.

(1982): Changes in joint laxity occurring during pregnancy. Annals of the Rheumatic Diseases. pregn: 126 - 128 .

Clapp J.F. (2006): Influence of endurance exercise and diet on human placental development and fetal growth. Placenta. Jul;27(67):527-34. DOI: 10.1016/j.placenta.2005.07.010 Dehghan F., Haerian B.S., Muniandy S. Yusof A., Dragoo J.L., and Salleh N. (2014): The effect of relaxin on the musculoskeletal system. Scandinavian Journal of Medicine \& Science in Sports. Aug; 24(4):220-229. doi: 10.1111/ sms.12149

Dunning K., LeMasters G., Levin L., Bhattacharya A., Alterman T., Lordo K.A. (2003): Falls in workers during pregnancy: risk factors, job hazards, and high risk occupation American Journal of Industrial Medicine. Dec;44(6):664-72. DOI: 10.1002/ajim.10318 Forczek W. and Staszkiewicz R. (2012): Changes of kinematic gait parameters due to pregnancy. Acta of Bioengineering and Biomechanics. 14(4): 113-11

Foti, T., Davids J. R. and Bagley A. (2000): A biomechanical analysis of gait during pregnancy. Journal of Bone and Joint Surger. 82(5): $625-632$.

Garber C.E., Blissmer B., Deschenes M.R., Franklin B. A Lamonte MJ, De IM. Nieman D.C. Swain D.P. (2011): American College of Sports Medicine position stand. Quantity and quality of exercise for developing and maintaining cardiorespiratory, musculoskeletal, and ne romotor fitness in apparently healthy adults: guidance for prescribing exercise. Medicine \& Science in Sports \& Exercise. Jul;43(7):1334-59. doi: 10.1249/MSS.0b013e318213fefb.

Gutke A., Ostgaard H.C. and Oberg B. (2008): Predicting persistent pregnancy-related low back pain. May;33(12):386-93. DOI: 10.1097/BRS 0 . 013 , 33(12):386-9.

Herring S.J., Platek D.N., Elliott P., Riley L.E., Stuebe A.M., Oken E. (2010): Addressing obesity in pregnancy: what do obstetric providors recommend? Journal of Women's Health (Larchmt). Jan;19(1):65-70. doi: 10.1089/

Hopkins S.A. and Artal R. (2013): Womens Health (Lond). The role of exercise in reducing the risks of gestational diabetes mellitus. Nov; 9(6):569-81. DOI: 10.2217/whe.13.52

Hopkins S.A. and Cutfield W.S. (2011): Exercise in pregnancy: weighing up the longterm impact on the next generation. Exercise and Sport Sciences Review. 39(3):120-127. doi 10.1097/JES.0b013e31821a5527

Huang T.H. Lin S.C., Ho C.S., Yu C.Y. and Chou Y.L. (2002): The gait analysis of pregnant women. Biomedical Engineering- Applications, Basis \& Communications. 14(2):

Karzel R.P. and Friedman M.J. (1991): Orthopedic injuries in pregnancy. In: Artal R, Wiswell RA, Drinkwater BL, eds. Exercise in pregnancy. 2nd ed. Baltimore: Williams and Wilkins.

Kramer M.S. and McDonald S.W. (2006): Aerobic exercise for women during pregnancy Juch (3): Database Systematic Reviews. 2006 CD000180.pub2

MacLennan A.H., Nicolson R., Green R.C.,
Bath M. (1986): Serum relaxin and pelvic pain of pregnancy. Lancet. Aug.2 (8501):243-5. DOI: 10.1016/S0140-6736(86)92069-6

Morris S.N., Johnson N.R. (2005): Exercise during pregnancy: a critical appraisa of the literature. Journal of Reproductive Medicine. 50:181-88. DOI:10.1590/S0102 $311 \times 2008001600006$

Moyer C., Livingston J., Fang X., and

May LE. (2015): Influence of exercise mode on pregnancy outcomes: enhanced by Mom poject. BMC Pregnancy and Childbirth. 15:133. DOI:10.1186/s12884-015-0556-6

Pate R.R., Pratt M., Blair S.N. (1995): A recommendation from the Centers for Disease Control and Prevention and the American College of Sports Medicine. JAMA. 273:402-7. doi:10.1001/jama.1995.03520290054029

Price B., Amini S. and Kappeler K. (2012): Exercise in pregnancy: effect on fitness and obstetric outcomes - a randomized trial. Medicine and Science in Sports \& Exercise. 44:2263-69. doi: 10.1249/MSS.0b013e318267ad67

Rodacki C.L., Fowler N.E., Rodacki A.L. and Birch K. (2003): Stature loss and recovery in pregnant women with and without low back pain. Archives of Physical Medicine and Rehabilitation. 84(4):507-512.

Rousham E., Clarke P. and Gross H. (2006): Significant changes in physical activity amon pregnant women in the UK as assessed by accelerometry and self-reported activity. European Journa doi:10.1038/sj.ejcn.1602329

Royal College of Obstetricians and

Szerző: Ressinka Judit

Title: Personal Trainer, Strength \& Conditioning Coach Munkahelye: Myhealthcare Clinic, Wandsworth Webpage: www.movepainfree.co.uk E-mail cime: juditressinkapt@gmail.com Főbb kutatási területei: fájdalommentes mozgás
Gynaecologists. RCOG Statement No. 4: Exere in Pregnancy. Available at www.rcog. Schoenfeld B. (2011):Resistance Training During Pregnancy: Safe and Effective Program Design. Strength \& Conditioning Journal. 33(5):67-75. DOI: 10.1519/SSC.0b013e$31822 \mathrm{ec} 2 \mathrm{~d} 8$

Shub A., Huning E.Y., Campbell K.J., McCarthy E.A. (2013): Pregnant women's knowledge of weight, weight gain, complications of obesity and weight management strategies in pregnancy. BMC Research Notes. 18(6):278. do:

12): Ca K., Shimizu K. and Imura M. third trimester Journal of Physical Therapy Science. Jun 27(6): 1813-1817. doi:10.1589/

Wang S.M., Dezinno P. Maranet I. (2004): \& AOG 0000129403.54061 .

Whitcome K.K., Shapiro L.J. and Lieberman D.E. (2007): Fetal load and the evolution of lumbar lordosis in bipedal hominins. Nature.

World Health Organization. Global health. Gendations on physical activity fo at: wwwint/dietphysicalactivity/publications/9789241599979/en.

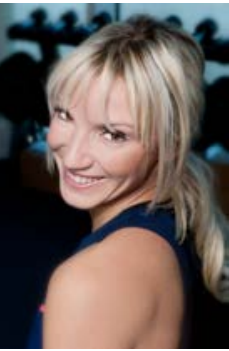

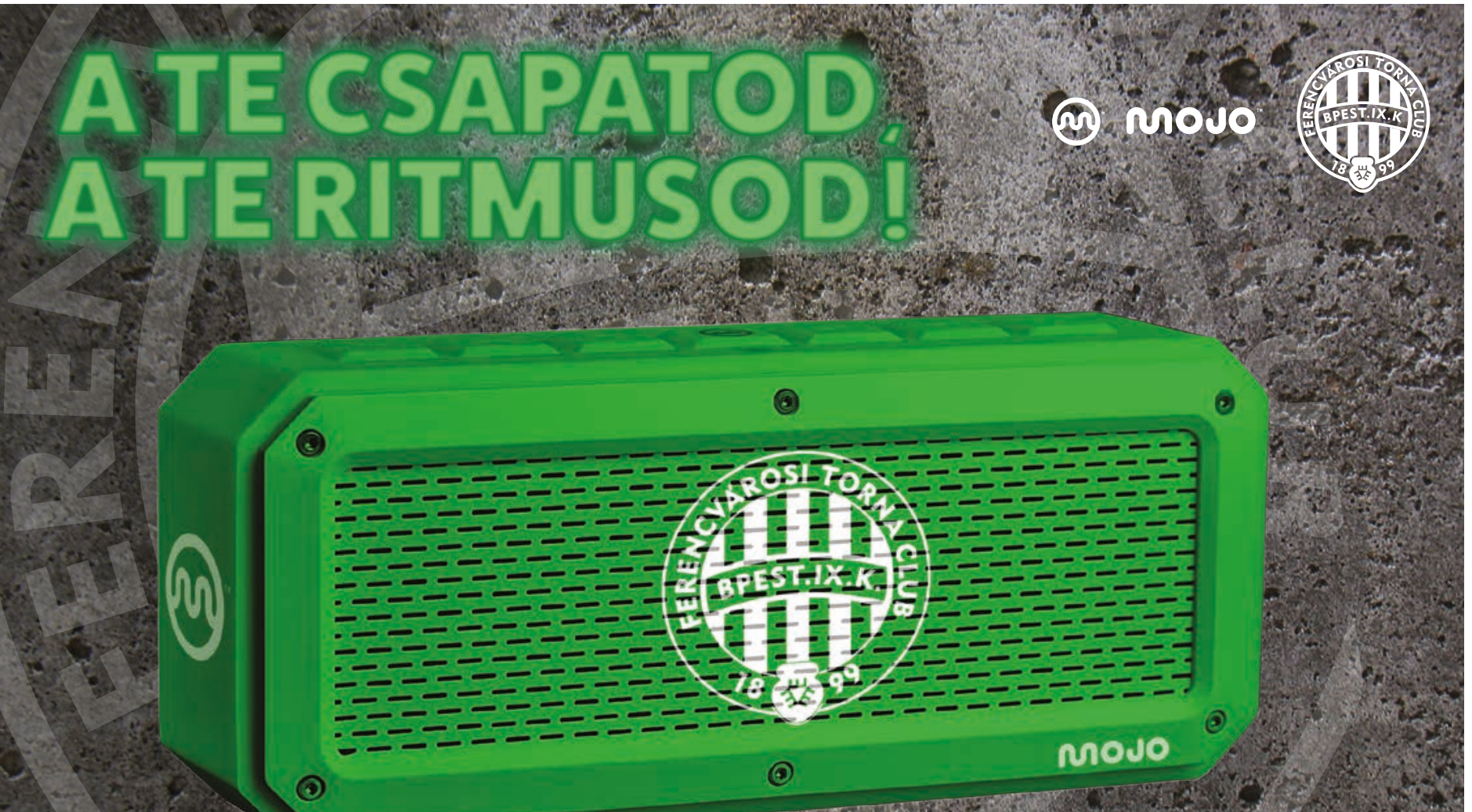

FRADI X MOJO BUZZ BLUETOOTH HANGSZÓRÓ

Megvásárolható: Fradishop shop.fradi.hu

(8) Bluetooth 